\title{
Validação Exploratória e Confirmatória da Escala de Resiliência Connor-Davidson (CD-RISC-10) numa Amostra de Inscritos em Centros de Emprego
}

\section{Exploratory and Confirmatory Validation of the Connor- Davidson Resilience Scale (CD-RISC-10) in a Sample of Individuals Registered in Job Centers}

\author{
Maria Helena ALMEIDA $\rrbracket^{1}$, Sara DIAS ${ }^{2,3}$, Miguel XAVIER ${ }^{4}$, Jorge TORGAL ${ }^{1}$ \\ Acta Med Port 2020 Feb;33(2):124-132 - https://doi.org/10.20344/amp.12243
}

\section{RESUMO}

Introdução: Bonanno definiu resiliência como a capacidade do indivíduo em lidar com os eventos traumáticos, nomeadamente a capacidade de manter os níveis de funcionamento físico e psicológico relativamente estáveis. O objetivo do estudo foi avaliar as propriedades psicométricas da escala de resiliência Connor-Davidson de 10 itens. A escala resumida apresenta boas qualidades psicométricas ( $\alpha$ Cronbach - 0,85) quando comparada com a escala longa ( 25 itens) (a Cronbach - 0,89).

Material e Métodos: Obtivemos uma amostra de conveniência de inscritos em centros de emprego que foi aleatoriamente dividida em duas subamostras (amostra $1=5127$ e amostra $2=5134$ ). Aplicámos a análise de componentes principais na amostra 1 . Com a amostra 2 procedeu-se à análise fatorial confirmatória usando o método da máxima verosimilhança. Usámos a análise multigrupo para a comparação do modelo fatorial entre os géneros e a situação profissional. Efetuámos a análise convergente e divergente através da análise de correlação de Pearson.

Resultados: A análise de componentes principais extraiu um fator com uma variância explicada de $53,97 \%$. A análise fatorial confirmatória comprovou a unidimensionalidade da escala. Confirmámos a invariância entre géneros e situação profissional. A escala de resiliência Connor-Davidson de 10 itens correlacionou-se positivamente com as medidas da escala SF-36 e negativamente com a escala GHQ-12.

Discussão: A análise exploratória e confirmatória corroborou a unidimensionalidade e boas propriedades psicométricas, semelhantes à escala original de 10 itens.

Conclusão: Trata-se do primeiro estudo de validação desta escala na população portuguesa (Portugal), de acordo com a pesquisa efetuada. Os seus resultados são muito satisfatórios, recomendando-se o seu uso na população portuguesa.

Palavras-chave: Inquéritos e Questionários; Portugal; Psicometria; Resiliência Psicológica

\section{ABSTRACT}

Introduction: Bonanno defined resilience as the individual's capacity to cope with traumatic events, namely, the ability to maintain the levels of physical and psychological functioning relatively stable. The aim of this study was to evaluate the psychometric properties of 10 -item Connor-Davidson resilience scale. The Connor-Davidson resilience scale short version has good psychometric qualities (Cronbach $\alpha-0.85$ ) when compared with the long version (Cronbach $\alpha-0.89$ ).

Material and Methods: We obtained a convenience sample of individuals registered in job centers which was randomly divided into two subsamples (sample $1=5127$ and sample $2=5114$ ). We applied the principal component analysis in sub-sample $\mathrm{n} 1$. With subsample $\mathrm{n} 2$ a confirmatory factorial analysis was performed using the maximum likelihood method. We used the multigroup analysis to compare the factorial model between genders and professional situation. We performed a convergent and discriminant analysis through Pearson's correlation analysis.

Results: The principal component analysis extracted one factor with an explained variance of $53.97 \%$. The confirmatory factor analysis confirmed the unidimensionality of the scale. The invariance between genders and professional situation was confirmed. The ConnorDavidson resilience scale correlated positively with the SF-36 scale and negatively with the GHQ-12 scale.

Discussion: The exploratory and confirmatory analysis revealed one dimension, and good psychometric properties, which is similar to the original 10 -item scale.

Conclusion: To our knowledge, this is the first validation study of this scale among the Portuguese population (Portugal). Its results are highly satisfactory, and its use is recommended among the Portuguese population.

Keywords: Portugal; Psychometrics; Resilience, Psychological; Surveys and Questionnaires

\section{INTRODUÇÃO}

Bonanno definiu resiliência como a capacidade do indivíduo em lidar com os eventos traumáticos, nomeadamente, a capacidade de manter os níveis de funcionamento físico e psicológico relativamente estáveis. ${ }^{1} \mathrm{~A}$ capacidade de o indivíduo se adaptar aos eventos negativos e à adversidade são hoje essenciais como forma de os ultrapassar com sucesso e superá-los dinamicamente. Os sujeitos resilientes são capazes de encontrar meios e recursos que lhes

1. Unidade Curricular de Saúde Publica, Epidemiologia e Bioestatística. NOVA Medical School - Faculdade de Ciências Médicas. Universidade NOVA de Lisboa. Lisboa. Portugal. 2. Center for Innovative Care and Health Technology (ciTechCare). Health Sciences School. Instituto Politécnico de Leiria. Leiria. Portugal.

3. EpiDoc Unit - Centro de Estudo de Doenças Crónicas (CEDOC). NOVA Medical School - Faculdade de Ciências Médicas. Universidade NOVA de Lisboa. Lisboa. Portugal.

4. Departamento de Saúde Mental. NOVA Medical School - Faculdade de Ciências Médicas. Universidade NOVA de Lisboa. Lisboa. Portugal.

$\square$ Autor correspondente: Maria Helena Almeida. mh09almeida@gmail.com

Recebido: 25 de abril de 2019 - Aceite: 30 de agosto de 2019 | Copyright @ Ordem dos Médicos 2020 
possibilitam agir positivamente e alcançar o equilíbrio sem disfunção maior. Experiências passadas feitas de sucessos e insucessos são oportunidades de fortalecimento, assim como uma adversidade superada positivamente pode ser relatada como um episódio de bem-estar. ${ }^{2}$ Fatores biológicos, genéticos, sociais e culturais parecem alterar a resiliência, pelo que o processo de resiliência e as estratégias de coping podem ser desenvolvidas e trabalhadas a favor de um melhor bem-estar e a bem da saúde mental. Estão de acordo com este princípio os autores Windle, Bennet \& Noyes ao definirem resiliência como "the process of negotiating, managing and adapting to significant sources of stress or trauma". ${ }^{3}$

O nível de resiliência parece variar de acordo com o género, a idade, a cultura, o contexto e o tempo em que decorre, alterando-se no decurso de vida de cada sujeito. ${ }^{4}$ A presença de stressores é uma constante e a capacidade de cada um em lidar com estes, depende da adaptação (ou não) a fenómenos disruptivos prévios. ${ }^{5,6}$ Contudo desconhece-se se a resiliência é um determinante da resposta ao stress, ou constitui um efeito da exposição ao stressor ou um processo bidirecional. ${ }^{7}$

King et al demonstraram que a resiliência é um forte preditor contra o desenvolvimento da síndroma pós-traumática crónica. ${ }^{8}$ Campbell-Sills \& Stein comprovaram que a resiliência (usando a escala Connor-Davidson - CD-RISC 10 itens) moderava a relação entre a existência passada de maus tratos e a presença de sintomatologia psiquiátrica. ${ }^{9}$ Os indivíduos que sofreram mais maus tratos apresentavam scores mais elevados de sintomatologia psiquiátrica comparativamente com aqueles que tinham sofrido menos maus tratos. Melhores níveis de resiliência encontram-se associados a melhores níveis de saúde mental (depressão e ansiedade). ${ }^{10,11}$

Alguns autores consideram não existir um teste de resiliência gold standard, mas ainda assim classificaram como sendo os melhores instrumentos o Resilience Scale for Adults (RSA), a escala CD-RISC de Connor-Davidson ${ }^{7,9}$ e o Brief Resilience Scale. ${ }^{3}$ Cada vez mais estes instrumentos estão a ser aplicados na medição das competências individuais de coping e na identificação de necessidades em saúde, entre grupos vulneráveis ${ }^{12-14}$ ou sujeitos a situações de trauma. ${ }^{15}$ Tem ainda sido usado como um instrumento de medida para discriminar grupos de risco que necessitem de intervenções precoces ${ }^{16} \mathrm{e}$, como marcador para detetar diferenças após intervenções terapêuticas, nomeadamente farmacológicas. . $^{71}$

Apesar de Connor \& Davidson terem originalmente abordado a multidimensionalidade da escala original de 25 itens, Campbell-Sills et al (2007) analisaram a escala em duas populações de jovens americanos e propuseram um modelo resumido unidimensional de 10 itens com boas propriedades psicométricas. ${ }^{9} \mathrm{~A}$ versão resumida apresentou tão boas propriedades psicométricas ( $\alpha$ Cronbach - 0,85) como a versão longa ( $\alpha$ Cronbach - 0,89). ${ }^{7,9}$ Os autores acreditam que a versão curta captura as caraterísticas principais da resiliência, uma vez que a sua unidimensio- nalidade está altamente correlacionada com os scores do instrumento original $(r=0,92) .{ }^{9}$

Outros investigadores têm corroborado a sua unidimensionalidade, as suas boas qualidades psicométricas e a sua facilidade de aplicação em populações, culturas e contextos diferentes. ${ }^{17,18,19-29}$ Quanto à análise de invariância da escala CD-RISC, entre grupos, ainda são escassos os trabalhos de investigação. Burns et al (2010) confirmaram, numa coorte de australianos a invariância configuracional e métrica da escala CD-RISC 25 entre géneros, no grupo etário dos 20 aos 24 anos. ${ }^{17}$ Uns anos mais tarde (2015), no seguimento do mesmo estudo de coorte, Liu et al evidenciaram no mesmo modelo unidimensional a invariância configuracional entre géneros em cada uma das três coortes estudadas (28 - 32; 48 - 52; 68 - 72 anos) e entre as coortes. No entanto a invariância de medida da escala só foi parcialmente demonstrada. ${ }^{30}$

O contexto de trabalho tem um forte impacto na vida das pessoas. A perda de emprego é frequentemente citado como uma das 10 mais nefastas experiências humanas, à semelhança do divórcio ou morte do conjuge. ${ }^{31}$ São escassos os trabalhos que estudam resiliência em adultos e especificamente associado a contexto de trabalho ou ao desemprego. O estudo de Moorhouse et al evidenciou numa amostra de desempregados que níveis mais baixos de depressão se encontravam associados a níveis mais elevados de resiliência. ${ }^{32} \mathrm{O}$ mesmo estudo revelou ainda que níveis elevados de resiliência associavam-se a valores mais baixos de depressão mesmo para desempregados de longa duração à procura de trabalho. Também Slebarska et al demonstraram que os desempregados à procura de emprego que apresentavam melhores níveis de suporte social apresentavam melhores níveis de resiliência. ${ }^{33}$

Em contexto de trabalho identificaram-se quatro estu dos de validação psicométrica da escala de CD-RISC 10 itens, nomeadamente três trabalhos com amostras de empregados e uma de desempregados..$^{23,25,26,34}$ No Brasil, Lopes et al procedeu à validação exploratória da escala de 10 itens numa amostra de 463 trabalhadores de diversos sectores profissionais. Obtiveram uma escala de uma única dimensão que explicou $38 \%$ da variância total, e uma fiabilidade de $0,82 .{ }^{25}$ Sánchez et al avaliaram as propriedades psicométricas de duas amostras espanholas de empregados usando a análise exploratória e confirmatória. $\mathrm{Na}$ primeira amostra ( $n=386$ ) obtiveram uma medida unidimensional que explicava $55,8 \%$ da variância e uma consistência interna de 0,87 . Na segunda amostra $(n=238)$ verificaram que os índices de ajustamento eram todos adequados corroborando as boas propriedades psicométricas da escala. ${ }^{23}$ Também num estudo de validação confirmatória da escala CD-RISC 10 itens num grupo de desempregados espanhóis da região de Múrcia $(n=223)$, Valera et al corroboraram uma configuração unifatorial, uma fiabilidade de 0,87 e bons índices de ajustamento. ${ }^{26}$ Mais recentemente uma amostra de auditores financeiros americanos $(n=390)$ a trabalhar no sector público, demonstrou as boas qualidades psicométricas da escala apesar de evidenciar um 
modelo de dois fatores. ${ }^{34}$

O presente estudo teve como objetivo analisar as propriedades psicométricas da escala CD-RISC 10 itens numa população portuguesa de inscritos em centros de emprego da Região de Lisboa e Vale do Tejo. A escala original CD-RISC de Connor-Davidson (25 itens) ${ }^{7}$ foi traduzida e validada para português ${ }^{35}$ e aprovada pelos autores Connor \& Davidson. ${ }^{36}$

\section{MATERIAL E MÉTODOS}

Extraíram-se duas amostras aleatórias (amostra $1=$ 5127 e amostra 2 = 5134) a partir de uma amostra de conveniência $(n=20658)$ de inscritos em centros de emprego da Região de Lisboa de Vale do Tejo, obtida através de um questionário via webmail (SurveyMonkey ${ }^{\circledR}$ ) entre dezembro de 2015 e março de 2017. Os resultados das variáveis selecionadas foram todas recolhidas pelo mesmo questionário via webmail no mesmo momento, incluindo as escalas CD-RISC10, SF-36v2 e a GHQ-12. A primeira amostra foi usada para a análise fatorial exploratória e a segunda amostra para a análise fatorial confirmatória. No âmbito deste estudo, para além das escalas, descritas a seguir, incluíram-se variáveis sociodemográficas (género, idade, estado civil, escolaridade, situação profissional e tempo de desemprego), para uma breve análise descritiva da amostra.

Para a análise sumaria dos dados, calculámos as médias e desvio-padrão (DP) para as variáveis quantitativas e as frequências absolutas e relativas para as variáveis qualitativas. Usámos os testes $\chi^{2}$ e $t$ de Student na análise entre subamostras. Usou-se o teste Kruskall-Wallis na avaliação das diferenças dos valores medianos entre categorias (género e situação profissional). Para a realização dos testes usámos o software SPSS ${ }^{\bullet}$ v. 23.

A escala CD-RISC contém cinco possíveis respostas, desde 'nada verdadeira' (zero pontos) até 'totalmente verdadeira' (quatro pontos) ${ }^{9,36} \mathrm{O}$ valor global pode atingir o máximo de 40 pontos. Quanto mais elevada a pontuação, maior o nível de resiliência. Analisámos a fiabilidade da escala usando o teste a Cronbach. Estudámos a dimensionalidade da escala, usando a matriz de correlações entre cada item e, cada item com a escala global. Considerámos correlações fortes acima de 0,5, moderadas entre 0,3 e 0,5, fracas inferiores a $0,3 .{ }^{37}$ Utilizámos a análise das componentes principais (ACP) com rotação Varimax para determinar o número de dimensões da escala. Usámos o critério Kaiser na extração dos componentes principais com valor próprio superior a um. Para avaliar a adequação do modelo usámos o teste de esfericidade de Bartlett e o critério de Kaiser-Meyer-Olkin (KMO).

Para a análise fatorial confirmatória usámos o software AMOS v. 24 (SPSS ${ }^{\odot}$ ) e o método da máxima verosimilhança. Para determinar a qualidade do ajustamento global do modelo fatorial empregámos os seguintes índices: razão do qui-quadrado pelos graus de liberdade ( $\left.\chi^{2} / \mathrm{df}\right)$, Godness-of-Fit Index (GFI), Comparative Fit Index (CFI), Root Mean Square Error of Aproximation (RMSEA), Standardized Root
Mean Square Residual (SRMSR) e Modified Expected Cross Validation (MECVI). Para os índices GFI, CFI considerámos um bom ajustamento para valores acima de 0,90 e muito bom acima de 0,95 . Hu et al recomendam valores próximos de 0,95 para CFI e inferiores a 0,08 para SRMR. ${ }^{38}$ Browne et al consideram para RMSEA valores aceitáveis entre 0,05 e $0,08 .{ }^{39}$ Para MECVI não existe um valor adequado mas o melhor modelo será aquele que apresentar menores valores. Por fim, utilizámos a análise multigrupo para avaliar a invariância do modelo entre género e situação ocupacional (desempregado, empregado e outras situações). Para efeito de comparação dos modelos usámos - $\triangle \mathrm{CFI}$ (difference between comparative fit index) entre grupos como proposto por Cheung et al. ${ }^{40}$ Segundo estes autores, uma redução do $\Delta \mathrm{CFI}$ superior a $-0,01(\Delta \mathrm{CFI}>$ $-0,01)$ é indicador da não invariância do modelo.

Para testar a validade convergente e divergente da escala utilizámos a correlação de Pearson com as seguintes dimensões: Short Form 36 Health Survey Instrument (SF-36v2), como uma medida de avaliação da perceção do estado de saúde, a escala General Health Questionnaire de 12 itens (GHQ-12) e o índice de massa corporal (IMC). ${ }^{41}$ Calculámos previamente as subescalas e as componentes física e mental da versão portuguesa da escala SF-36v2.42 $O$ valor mais elevado corresponde a uma melhor perceção da saúde. Quanto à escala GHQ-12, esta tem sido usada como medição de perturbações psiquiátricas minor ou como medição de distress psicológico em população geral. ${ }^{43} \mathrm{~A}$ versão portuguesa da GHQ-12 itens foi traduzida e validada por McIntery et all. ${ }^{44}$ Usámos a pontuação original de Goldberg que consiste em aplicar zero às duas primeiras respostas e 1 às restantes respostas $(0-0-1-1) \cdot{ }^{43}$ O somatório de cada questão pode totalizar um mínimo de zero e um máximo de 12 pontos. Quanto mais elevado o valor, mais elevado o nível de distress psicológico. Para a análise estatística utilizámos o nível de significância usual, $5 \%$. O estudo foi aprovado pela Comissão de Ética da Nova Medical School, Universidade Nova de Lisboa (Projeto n. ${ }^{\circ}$ 33/2014/CEFCM) e pela Comissão Nacional de Proteção de Dados (Deliberação n. ${ }^{\circ}$ 1614/2015 CNPD).

\section{RESULTADOS}

Apresenta-se uma análise sumária das duas amostras em estudo na Tabela 1. As amostras (amostra 1 e amostra 2) eram constituídas maioritariamente por mulheres (62,59\% e 62,27\%), e mais jovens comparativamente com os homens (36,95 e 36,73 anos vs 42,71 e 42,62 anos). Setenta e dois por cento estavam desempregados; $64 \%$ encontravam-se desempregados há mais de 12 meses. Ambas as amostras apresentavam níveis de resiliência muito semelhantes [amostra 1 - 25,94 (DP 7,16); amostra $2-25,93$ (DP 7,12)]. As mulheres apresentavam valores de resiliência significativamente inferiores aos homens em ambas as amostras (amostra 1, $p<0,01$; amostra 2, $p$ $<0,01$ ) (dados não apresentados na tabela 1). Relativamente à situação profissional, na amostra 1 os desempregados apresentavam níveis de resiliência (mediana $=26$ ) 
Tabela 1 - Descrição sumaria das duas amostras

\begin{tabular}{|c|c|c|c|}
\hline & Amostra 1 & Amostra 2 & $p$ \\
\hline Amostra (n) | (\%) & 5127 & 5134 & \multirow{3}{*}{$0,740^{*}$} \\
\hline Homens & $1918(37,41)$ & $1937(37,73)$ & \\
\hline Mulheres & $3209(62,59)$ & $3197(62,27)$ & \\
\hline Idade | média ( DP) & $39,10(12,07)$ & $38,94(12,11)$ & $0,547^{* *}$ \\
\hline Homem & $42,71(12,60)$ & $42,62(12,62)$ & $0,680^{* *}$ \\
\hline Mulher & $36,95(11,19)$ & $36,73(11,23)$ & $0,591^{\text {** }}$ \\
\hline \multicolumn{4}{|l|}{ Estado Civil | (\%) } \\
\hline Solteiros & $2368(46,19)$ & $2448(47,68)$ & \multirow{3}{*}{$0,199^{*}$} \\
\hline Casados/união & $2053(40,04)$ & $2029(39,52)$ & \\
\hline Divorciados/separados & $706(13,77)$ & $657(12,80)$ & \\
\hline \multicolumn{4}{|l|}{ Ensino (n) | (\%) } \\
\hline Básico & $471(9,19)$ & $467(9,10)$ & \multirow{3}{*}{$0,699^{*}$} \\
\hline Secundário & $2109(41,13)$ & $2074(40,40)$ & \\
\hline Universitário & $2547(49,68)$ & $2593(50,50)$ & \\
\hline \multicolumn{4}{|l|}{ Sit. Ocupacional | (\%) } \\
\hline Desempregado & $3694(72,05)$ & $3686(71,80)$ & \multirow{3}{*}{$0,642^{*}$} \\
\hline Empregado & $740(14,43)$ & $724(14,10)$ & \\
\hline Outra situação & $693(13,52)$ & $724(14,10)$ & \\
\hline \multicolumn{4}{|l|}{ Duração desemprego (n) | (\%) } \\
\hline$<12$ meses & $1824(35,58)$ & $1848(36,00)$ & \multirow{2}{*}{$0,658^{*}$} \\
\hline$\geq 12$ meses & $3303(64,42)$ & $3286(64,00)$ & \\
\hline \multicolumn{4}{|l|}{ Resiliência | média (SD) } \\
\hline Global & $25,94(7,16)$ & $25,93(7,12)$ & $0,657^{* *}$ \\
\hline Homem & $26,62(7,04)$ & $26,52(6,95)$ & $0,975^{\text {** }}$ \\
\hline Mulher & $25,53(7,20)$ & $25,57(7,20)$ & $0,597^{* *}$ \\
\hline Desempregado & $25,74(7,24)$ & $25,82(7,11)$ & $0,278^{* *}$ \\
\hline Empregado & $26,19(6,76)$ & $26,23(6,68)$ & $0,805^{* *}$ \\
\hline Outras situações profissionais & $26,69(7,08)$ & $26,22(7,54)$ & $0,121^{* *}$ \\
\hline
\end{tabular}

${ }^{*}: \chi^{2} ;{ }^{* *} t$ test: Testes entre amostras

estatisticamente inferiores ao grupo - outras situações profissionais não caracterizadas (mediana $=27)(p=0,02)$ mas sem significado estatístico relativamente com o grupo dos recentemente empregados (mediana $=27$ ) obtida através do teste de Kruskall-Wallis e a análise de pares de grupos usando o procedimento de Dunn com a correção de Bonferroni. Na amostra 2 não se verificou diferenças estatisticamente diferentes entre os três grupos (dados não apresentados na Tabela 1).

A escala CD-RISC aplicada à amostra 1 ( $n=5127)$ apresentou uma pontuação média de 25,94 (DP 7,16). Procedeu-se à avaliação da dimensionalidade da escala, analisando a matriz de correlações entre cada item e cada item com a escala global. Espera-se que as correlações atinjam valores iguais ou acima de $0,40 .{ }^{45}$ Quase todas as correlações encontravam-se acima de 0,40 e todas eram estatisticamente significativas $(p<0,01)$ (Tabela 2$)$. As correlações item - total da escala variaram entre os valores 0,67 e 0,81 (Tabela 2).

Obtivemos na amostra 1 para a escala CD-RISC uma consistência interna de 0,91 para homens e 0,90 para muIheres. Analisámos a consistência interna para os grupos de desempregados $(0,91)$, empregados $(0,89)$ e outras situações profissionais $(0,90)$ respetivamente; todos os valores apresentavam correlações adequadas. ${ }^{46}$

$\mathrm{Na}$ análise de componentes principais (ACP) o teste de esfericidade de Bartlett $(p<0,001)$ e a medida de KMO $(\mathrm{KMO}=0,934)$ confirmaram que o modelo era adequado aos dados. Foi extraída uma componente pelo critério de Kaiser e confirmada pelo scree-plot. Esta solução de uma dimensão apresentou uma variância explicada de 53,97\%. Os valores dos pesos fatoriais variaram entre 0,660 e 0,819 e as comunalidades entre 0,435 e 0,672 (Tabela 3). ${ }^{47}$

Todas as correlações foram estatisticamente significativas $(p<0,01)$, exceto o IMC que não apresentou correlação com a escala CD-RISC ( $r=-0,007, p=0,592)$ (Tabela 4). A escala CD-RISC apresentou uma correlação positiva e estatisticamente significativa com as subescalas e componentes física e mental da escala SF-36. As correlações mais fortes ocorreram com a componente Mental 
Tabela 2 - Matriz de correlação Pearson dos itens da escala CD-RISC 10 itens

\begin{tabular}{|c|c|c|c|c|c|c|c|c|c|c|}
\hline & CDRISC1 & CDRISC2 & CDRISC3 & CDRISC4 & CDRISC5 & CDRISC6 & CDRISC7 & CDRISC8 & CDRISC9 & CDRISC10 \\
\hline CDRISC1 & 1 & & & & & & & & & \\
\hline CDRISC2 & $0,608^{*}$ & 1 & & & & & & & & \\
\hline CDRISC3 & $0,411^{*}$ & $0,492^{*}$ & 1 & & & & & & & \\
\hline CDRISC4 & $0,380^{*}$ & $0,467^{*}$ & $0,482^{*}$ & 1 & & & & & & \\
\hline CDRISC5 & $0,420^{*}$ & $0,445^{*}$ & $0,383^{*}$ & $0,424^{*}$ & 1 & & & & & \\
\hline CDRISC6 & $0,499^{*}$ & $0,525^{*}$ & $0,442^{*}$ & $0,480^{*}$ & $0,504^{*}$ & 1 & & & & \\
\hline CDRISC7 & $0,452^{*}$ & $0,472^{*}$ & $0,394^{*}$ & $0,561^{*}$ & $0,460^{*}$ & $0,557^{*}$ & 1 & & & \\
\hline CDRISC8 & $0,402^{*}$ & $0,433^{*}$ & $0,391^{*}$ & $0,428^{*}$ & $0,412^{*}$ & $0,523^{*}$ & $0,537^{*}$ & 1 & & \\
\hline CDRISC9 & $0,512^{*}$ & $0,551^{*}$ & $0,460^{*}$ & $0,481^{*}$ & $0,470^{*}$ & $0,664^{*}$ & $0,562^{*}$ & $0,598^{*}$ & 1 & \\
\hline CDRISC10 & $0,460^{*}$ & $0,546^{*}$ & $0,450^{*}$ & $0,462^{*}$ & $0,467^{*}$ & $0,526^{*}$ & $0,518^{*}$ & $0,503^{*}$ & $0,644^{*}$ & 1 \\
\hline SUM_CDRISC & $0,687^{*}$ & $0,745^{*}$ & $0,674^{*}$ & $0,716^{*}$ & $0,679^{*}$ & $0,777^{\star}$ & $0,756^{*}$ & $0,719^{*}$ & $0,812^{*}$ & $0,764^{\star}$ \\
\hline
\end{tabular}

$(r=0,566, p<0,01)$, as subescalas Mental $(r=0,505, p$ $<0,01)$, Vitalidade $(r=0,528, p<0,01)$, Saúde Geral $(r$ $=0,465, p<0,01)$ e Funcionamento Social $(r=0,459, p$ $<0,01)$. A escala de resiliência correlacionou-se negativamente com a escala GHQ-12 $(r=-0,448, p<0,01)$ i.e. os indivíduos com níveis elevados de resiliência tendem a apresentar níveis baixos de distress psicológico. (Tabela 4).

Para a análise fatorial confirmatória utilizámos a segunda amostra constituida por 5134 indivíduos e o modelo unidimensional identificado. Os pesos fatoriais estandardizados estavam compreendidos entre 0,66 e 0,81 e a fiabilidade individual de todos os itens era superior a 0,25. Obtivemos os seguintes índices de ajustamento: $\chi^{2} / \mathrm{Df} p<$ 0,$001 ; \mathrm{GFI}=0,954 ; \mathrm{CFI}=0,950 ; \mathrm{RMSEA}=0,082 \mathrm{IC} 95 \%$ $(0,078 ; 0,086)$; SRMR =0,033; MECVI = 0,249 (Tabela 5). Uma vez que o modelo original apresentava índices adequados tendo em conta os limites aceitáveis recomendados por Browne et $a^{\beta 9}$ para valores de RMSEA (entre 0,05 e 0,08 ), prosseguiu-se para a análise de multigrupo para verificar a invariância do constructo relativamente ao género e à situação profissional.

Aplicando a mesma estrutura basal a ambos os grupos (homens e mulheres) achámos os seguintes índices de

Tabela 3 - Descrição da carga fatorial e respetivas comunalidades da escala CD-RISC

\begin{tabular}{lcc}
\hline Item & Peso fatorial & Comunalidades \\
\hline CDRISC1 & 0,701 & 0,491 \\
CDRISC2 & 0,756 & 0,572 \\
CDRISC3 & 0,660 & 0,435 \\
CDRISC4 & 0,701 & 0,491 \\
CDRISC5 & 0,675 & 0,455 \\
CDRISC6 & 0,786 & 0,617 \\
CDRISC7 & 0,754 & 0,568 \\
CDRISC8 & 0,713 & 0,509 \\
CDRISC9 & 0,819 & 0,672 \\
CDRISC10 & 0,766 & 0,586 \\
\hline
\end{tabular}

ajustamento $\mathrm{GFI}=0,951 ; \mathrm{CFI}=0,945 ; \mathrm{RMSEA}=0,061 ; \mathrm{IC}$ $95 \%(0,059 ; 0,062)$ exceto o $\chi^{2} / D f(p<0,001)$ (Tabela 6$)$. Há que ter em consideração que para grandes amostras este teste apresenta sempre valores estatisticamente significativos, não sendo o índice a ajustamento mais apropriado. ${ }^{48,49}$ Os índices de ajustamento do modelo fatorial entre homens e mulheres demonstraram a invariância configuracional (Tabela 5). Procedeu-se à análise da invariância métrica (pesos fatoriais fixos), invariância escalar (pesos fatoriais mais intercetos fixos) e invariância estrita (pesos fatoriais mais intercetos mais variâncias/covariâncias dos erros fixos). Os modelos de medida métrica, escalar e estrita entre homens e mulheres não apresentaram um ajustamento significativamente pior quando comparado com o modelo livre $(\Delta \mathrm{CFI}<-0,01)$ de acordo com o proposto por Cheung et al (Tabela 6). ${ }^{40}$

Os índices de ajustamento para o modelo fatorial entre as diferentes situações profissionais (empregados, desempregados e outras situações) demonstraram equivalência configuracional $(\mathrm{GFI}=0,951 ; \mathrm{CFI}=0,949$; $\mathrm{RMSEA}=0,048$; IC $95 \%[0,045 ; 0,050]$. Sequencialmente, procedemos à análise multigrupo impondo restrições para verificar a invariância entre os grupos com situações profissionais distintas. Verificou-se que as diversas restrições não pioraram o modelo de ajustamento dos grupos em análise $\Delta C F I<$ $-0,01)$ pelo que se pode afirmar a existência de invariância entre os grupos ocupacionais. Ficou demonstrada a invariância de medida forte dos dois modelos.

\section{DISCUSSÃO}

Este estudo teve como propósito proceder à validação exploratória e confirmatória da escala CD-RISC de 10 itens numa amostra de inscritos em centros de emprego da região de Lisboa e Vale do Tejo. O valor médio global obtido com a escala Connor-Davidson de 10 itens foi de 25,94 (DP $7,16)$, estando contido no intervalo dos valores encontrados na literatura. ${ }^{36}$ Esta escala foi anteriormente aplicada a uma população geral da cidade de Lisboa $(n=421)$ e obteve um valor médio de 29,3 (DP 5,7). ${ }^{35,36}$ Os autores da validação da escala de 10 itens (Campbell-Sills et al) 
Tabela 4 - Correlação entre a escala CD-RISC e outras dimensões

\begin{tabular}{|c|c|c|c|c|c|c|c|c|c|c|}
\hline & CDRISC & IMC & GHQ & $\begin{array}{l}\text { Saúde } \\
\text { Geral }\end{array}$ & Vitalidade & $\begin{array}{l}\text { Funcion. } \\
\text { Social }\end{array}$ & $\begin{array}{l}\text { Desemp. } \\
\text { Emocional }\end{array}$ & $\begin{array}{l}\text { Saúde } \\
\text { Mental }\end{array}$ & $\begin{array}{l}\text { Comp. } \\
\text { Físico }\end{array}$ & $\begin{array}{l}\text { Comp. } \\
\text { Mental }\end{array}$ \\
\hline CDRISC 10 itens & 1 & & & & & & & & & \\
\hline IMC & $-0,007$ & 1 & & & & & & & & \\
\hline GHQ 12 itens & $-0,448^{*}$ & $0,076^{*}$ & 1 & & & & & & & \\
\hline Subescala Saúde Geral ${ }^{\ddagger}$ & $0,465^{*}$ & $-0,129^{*}$ & $-0,443^{*}$ & 1 & & & & & & \\
\hline Subescala Vitalidade ${ }^{\ddagger}$ & $0,528^{*}$ & $-0,041^{*}$ & $-0,641^{*}$ & $0,572^{*}$ & 1 & & & & & \\
\hline $\begin{array}{l}\text { Subescala Funcionamento } \\
\text { Social }^{\ddagger}\end{array}$ & $0,459^{*}$ & $-0,011$ & $-0,633^{*}$ & $0,506^{*}$ & $0,697^{*}$ & 1 & & & & \\
\hline $\begin{array}{l}\text { Subescala Desempenho } \\
\text { Emocional }^{\ddagger}\end{array}$ & $0,427^{*}$ & $-0,027$ & $-0,519^{*}$ & $0,434^{*}$ & $0,584^{*}$ & $0,647^{*}$ & 1 & & & \\
\hline Subescala Saúde Mental ${ }^{\ddagger}$ & $0,505^{*}$ & $-0,021$ & $-0,636^{*}$ & $0,448^{*}$ & $0,639^{*}$ & $0,669^{*}$ & $0,587^{*}$ & 1 & & \\
\hline Componente Física ${ }^{\ddagger}$ & $0,416^{*}$ & $-0,167^{*}$ & $-0,442^{*}$ & $0,798^{*}$ & $0,591^{*}$ & $0,578^{*}$ & $0,569^{*}$ & $0,467^{*}$ & 1 & \\
\hline Componente Mental $^{\ddagger}$ & $0,566^{*}$ & $-0,034^{\dagger}$ & $-0,717^{*}$ & $0,581^{*}$ & $0,886^{*}$ & $0,851^{*}$ & $0,812^{*}$ & $0,842^{*}$ & $0,646^{*}$ & 1 \\
\hline
\end{tabular}

${ }^{*}: p<0,01 ;$ †: $p<0,05 ;$ : Subescalas e os componentes Sumário Físico e Mental da escala SF-36

Tabela 5 - Índices de ajustamento da escala unidimensional CD-RISC (Amostra $2 \mathrm{n}=5134$ )

\begin{tabular}{lccccccc}
\hline Descrição & $\chi^{2}$ IDf & $\boldsymbol{p}$ & GFI & CFI & RMSEA (IC) & SRMR & MECVI \\
\hline CD-RISC & $1235,520 / 35$ & 0,000 & 0,954 & 0,950 & $0,082(0,078-0,086)$ & 0,033 & 0,249 \\
\hline
\end{tabular}

Tabela 6 - Análise multigrupo. Medidas de ajustamento da escala CD-RISC entre género e entre situação profissional.

\begin{tabular}{|c|c|c|c|c|c|c|c|}
\hline & & $\chi^{2}$ & DF & $p$ & CFI & RMSEA & $\Delta \mathrm{CFI}$ \\
\hline \multirow{4}{*}{ 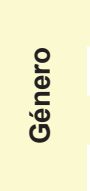 } & Modelo não restrito & 1235,520 & 35 & 0,000 & 0,950 & $0,082(0,078-0,086)$ & - \\
\hline & Modelo de medida métrica & 1437,689 & 79 & 0,730 & 0,945 & $0,058(0,055-0,061)$ & 0,000 \\
\hline & Modelo de medida escalar & 1534,722 & 89 & 0,000 & 0,941 & $0,056(0,054-0,059)$ & $-0,004$ \\
\hline & Modelo de medida estrita & 1535,170 & 90 & 0,504 & 0,941 & $0,056(0,054-0,059)$ & 0,000 \\
\hline \multirow{4}{*}{ 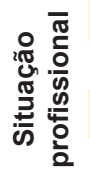 } & Modelo não restrito & 1330,766 & 105 & 0,000 & 0,949 & $0,048(0,045-0,050)$ & - \\
\hline & Modelo de medida métrica & 1361,427 & 123 & 0,000 & 0,949 & $0,044(0,042-0,046)$ & 0,000 \\
\hline & Modelo de medida escalar & 1469,310 & 143 & 0,000 & 0,945 & $0,043(0,041-0,045)$ & $-0,003$ \\
\hline & Modelo de medida estrita & 1479,483 & 145 & 0,000 & 0,945 & $0,042(0,040-0,044)$ & 0,000 \\
\hline
\end{tabular}

obtiveram um valor médio de 27,21 (DP 5,84) numa amostra de universitários, semelhante a outros trabalhos realizados com jovens universitários [Notário-Pacheco em Espanha - 27,41 (DP 6,36) - ou Aloba et al na Nigéria - 27,64 (DP 6,44)].9,20,50 Também Lopes et al analisaram em contexto ocupacional populações de trabalhadores brasileiros saudáveis, tendo obtido um valor médio de resiliência mais elevado (29,07 (DP 5,47) e Lauridsen et al numa amostra de profissionais de saúde dinamarqueses acharam um valor médio de 30,3 (DP 5,2). ${ }^{25,51}$ Identificamos um estudo de validação psicométrica da escala CD-RISC 10 itens numa população de desempregados espanhóis $(n=226)$. Contudo, não apresentavam o valor médio da resiliência. ${ }^{26}$ Estudos cujas amostras eram constituídas por indivíduos vítimas de um evento traumático apresentavam valores de resiliência mais baixos e mais próximos do aqui obtido [(amostra 1 - 25,94 (7,16); amostra 2 - 25,93 (DP 7,12)]. É o caso do estudo de resiliência em professores, vítimas de tremor de terra, de Wang Li et al [24,83 (DP 7,42)] e, o estudo, realizado por Shin et al, em operários expostos ao ácido hidrosulfúrico, [(26,25 (DP 7,64) para homens e 22,61 (DP 7,15) para mulheres)]. ${ }^{22,24}$ Os participantes do presente estudo eram indivíduos que sofreram um evento traumático i.e. perderam o emprego, pelo que já se esperava níveis de resiliência mais baixos. De acordo com os autores Connor \& Davidson, valores médios de resiliência entre 29,0 e 33,5 , entre 19,9 e 30,3 , e entre 19,6 e 25,8 têm sido encontrados na população geral, em indivíduos expostos a eventos traumáticos e, sujeitos com depressão e patologia psiquiátrica respetivamente. ${ }^{36}$ 
Verificámos que as mulheres apresentavam valores médios de resiliência inferiores $(25,53)$ aos apresentados pelos homens $(26,62)$. Este resultado foi evidenciado por outros investigadores tanto em indivíduos saudáveis como quem era exposto a acontecimentos traumáticos. ${ }^{20,22,50,52}$

A análise fatorial exploratória e confirmatória corroborou as boas propriedades psicométricas, e a unidimensionalidade da escala à semelhança do proposto pelos autores Campbell-Sills et al (2007), assim como noutros estudos em diferentes culturas, idades e contextos. ${ }^{20-25,28,52}$ Contudo alguns estudos evidenciaram mais de uma dimensão pelo que será necessário continuar a investigar noutras populações e contextos. ${ }^{34,50}$

A validade de critério da escala foi confirmada através da validade convergente e divergente. Relativamente ao distress psicológico medido através da escala GHQ-12, verificámos uma correlação negativa moderada $(r=-0,448, p$ $<0,01)$ no sentido teórico esperado e encontrado noutros trabalhos com desempregados e empregados espanhóis (Valera et al, $r=-0,490$; Soler et al, $r=-0,470) .{ }^{23,26}$ Também Smith et al verificaram uma correlação moderadamente negativa $(r=-0,449, p<0,001)$ entre a subescala de distress psicológico da perceived stress scale (PSS10) e a escala de CD-RISC10. ${ }^{34}$ Notário-Pacheco et al demonstraram em jovens universitários, que níveis reduzidos da perceção da saúde mental estavam associados a níveis reduzidos de resiliência. ${ }^{20}$ No nosso estudo as correlações mais fortes ocorreram com a componente Sumária Mental da escala SF-36 $(r=0,566, p<0,01)$, as subescalas Mental $(r=$ $0,505, p<0,01)$, Vitalidade $(r=0,528, p<0,01)$ e Funcionamento Social $(r=0,459, p<0,01)$, como esperado e sugerindo que a resiliência incorpora outras características. A subescala Desempenho Emocional $(r=0,427, p<$ $0,01)$ e a componente Sumária Física da escala SF-36 ( $r=$ $0,416, p<0,01)$ também apresentaram correlações moderadas e significativas. Também Lamond et al evidenciaram numa amostra de mulheres americanas uma correlação moderada entre a resiliência e a subescala Desempenho Emocional. ${ }^{53}$ Jeong et al numa amostra de bombeiros sul-coreanos verificou que as componentes Sumária Física e Mental (SF-36) apresentavam uma correlação moderada e significativa semelhante aos nossos resultados. ${ }^{54}$ Como era esperado e apontado por Goins et al, não existia correlação entre a resiliência e o IMC. ${ }^{28}$

Os pesos fatoriais encontrados através da análise confirmatória foram muito satisfatórios $(0,66$ a 0,82$)$, sendo ligeiramente superiores aos encontrados na escala original $(0,44$ - 0,74, Campbell-Sills et al, 2007) e noutros estudos, ${ }^{20,23,28,55}$ mas semelhantes a outros trabalhos. ${ }^{21,27}$

A análise confirmatória evidenciou índices de ajustamento bons e aceitáveis. O índice de ajustamento RMSEA encontrava-se no limite superior. Contudo, Browne et al, e McDonald et al, sugerem que valores de RMSEA próximos de 0,08 são aceitáveis. ${ }^{39,56}$ Algumas avaliações psicométricas da escala CD-RISC 10 itens têm evidenciado valores de RMSEA próximos ou acima de 0,08. ${ }^{17,21,28}$

Demonstrámos a invariância completa do constructo entre géneros à semelhança do encontrado em Campbell-Sills et a/ ${ }^{\rho}$ (2007). Também Burns et al (2010) provaram a invariância da escala entre géneros (configuracional e métrica), numa amostra de jovens australianos entre os 20 e os 24 anos. ${ }^{17}$ Mais tarde, González et al (2016), num estudo realizado em atletas americanos, corroboraram igualmente a invariância do modelo de 10 itens, entre os géneros. ${ }^{19} \mathrm{Re}$ centemente Smith et al (2018) demonstraram a invariância de constructo entre os géneros numa amostra de auditores financeiros americanos. ${ }^{34}$ Não nos foi possível identificar estudos que tenham analisado a invariância entre empregados e desempregados como descrito neste estudo. Realçamos neste trabalho a equivalência de constructo entre as três situações profissionais, sugerindo estabilidade nas características intrínsecas da escala, entre os grupos.

Um dos pontos fortes metodológicos deste estudo é a razão entre o número de participantes da amostra e o número de itens da escala, que consolida a estabilidade da estrutura fatorial unidimensional encontrada. ${ }^{57}$

Uma limitação do estudo é o seu desenho transversal, não permitindo concluir a validade preditiva entre a resiliência e as medidas analisadas. Só um estudo longitudinal nos permitiria avaliar se os indivíduos com características resilientes seriam capazes de enfrentar melhor o evento adverso comparativamente com os que não experienciaram o desemprego.

A amostra foi obtida por conveniência, na região de Lisboa e Vale do Tejo, em inscritos no IEFP, não podendo ser generalizada para a população que Ihe deu origem.

A escala CD-RISC é uma escala que simultaneamente mede traços de personalidade e a resposta a eventos stressores, i.e. tanto mede características que facilitam a resiliência como atesta as respostas adaptativas aos fenómenos adversos, o que constitui em si mesma a resiliência. ${ }^{16}$ A resiliência não é um constructo 'monocausal' pois é influenciada por outros fatores biopsicosociais, como ficou patente na aproximação com algumas medidas analisadas neste trabalho, pelo que este estudo apenas aborda uma característica de múltiplas outras que podem ser medidas. ${ }^{58}$

\section{CONCLUSÃO}

Este estudo permitiu verificar as boas qualidades psicométricas da escala CD-RISC 10 itens numa amostra portuguesa de inscritos no IEFP na região de Lisboa e Vale do Tejo evidenciando a mesma estrutura da escala original. Este estudo parece ser, da pesquisa efetuada, o primeiro trabalho de validação numa amostra da população portuguesa. Esta validação incidiu sobre desempregados e empregados portugueses demonstrando a mesma estabilidade psicométrica. Recomenda-se para o futuro que se aplique esta escala a outras populações, nomeadamente as que apresentem outros eventos adversos ou comorbilidades, e se procure avaliar as suas características psicométricas comparando com os dados agora divulgados. Julgamos que a escala CD-RISC 10 itens, simples e de aplicação fácil, é uma medida que contribuirá seguramente 
para uma melhor compreensão sobre a resiliência e o seu papel preditor na saúde mental.

\section{PROTECÇÃO DE PESSOAS E ANIMAIS}

Os autores declaram que os procedimentos seguidos estavam de acordo com os regulamentos estabelecidos pelos responsáveis da Comissão de Investigação Clínica e Ética e de acordo com a Declaração de Helsínquia da Associação Médica Mundial.

\section{REFERÊNCIAS}

1. Bonanno GA. Loss, trauma, and human resilience: have we underestimated the human capacity to thrive after extremely aversive events? Am Psychol. 2004;59:20-8.

2. Seery MD, Holman EA, Silver RC. Whatever does not kill us: cumulative lifetime adversity, vulnerability, and resilience. J Pers Soc Psychol. 2010;99:1025-41.

3. Windle G, Bennett KM, Noyes J. A methodological review of resilience measurement scales. Health Qual Life Outcomes. 2011;9:8.

4. Seligman M, Csikszentmihalyi M. Positive psychology: an introduction. Am Psychol. 2000;55:5-14.

5. Richardson G, Neiger B, Jensen S, Kumpfer K. The resiliency model. Health Educ. 1990;21:33-9.

6. Richardson G. The metatheory of resilience and resiliency. J Clin Psychol. 2002;58:307-21.

7. Connor KM, Davidson JR. Development of a new resilience scale: the Connor-Davidson Resilience Scale (CD-RISC). Depress Anxiety. 2003;18:76-82.

8. King LA, King DW, Fairbank JA, Keane TM, Adams GA. Resiliencerecovery factors in post-traumatic stress disorder among female and male Vietnam veterans: hardiness, postwar social support, and additional stressful life events. J Pers Soc Psychol. 1998;74:420-34.

9. Campbell-Sills L, Stein MB. Psychometric analysis and refinement of the Connor - Davidson resilience scale (CD-RISC): validation of a 10-item measure of resilience. J Trauma Stress. 2007;20:1019-28.

10. Rutter M. Psychosocial resilience and protective mechanisms. Am J Orthopsychiatry. 1987;57:316-31.

11. Pollack MH, Stein MB, Davidson JR, Ginsberg DL. New challenges for anxiety disorders: where treatment, reslience, and economic priority converge. CNS Spectr. 2004;9:1-4.

12. World Health Organization - Regional Office for Europe. Impact of Economic Crises on Mental Health. Geneve: WHO; 2011.

13. Smith-Osborne A, Whitehill Bolton K. Assessing resilience: a review of measures across the life course. J Evid Based Soc Work. 2013;10:11126.

14. Parkinson J, editor. Review of scales of positive mental health validated for use with adults in the UK: technical report. Edinburgh: Health Scotland; 2007.

15. Waysman M, Schwarzwald J, Solomon Z. Hardiness: an examination of its relationship with positive and negative long term changes following trauma. J Trauma Stress. 2001;14:531-48.

16. Serrano-Parra MD, Garrido-Aejar M, Notario-Pacheco B, BartoloméGutiérrez R, Solera-Martínez M, Martinez-Vizcaino V. Validez de la escala de Resiliencia de Connor- Davidson (CD-RISC) en una población de mayores entre 60 y 75 años. Int J Psychol Res. 2012;5:49-57.

17. Burns RA, Anstey KJ. The Connor-Davidson resilience scale (CDRISC): testing the invariance of a uni-dimensional resilience measure that is independent of positive and negative affect. Pers Individ Dif. 2010;48:527-31.

18. Gucciardi DF, Jackson B, Coulter TJ, Mallett CJ. The ConnorDavidson resilience scale (CD-RISC): dimensionality and age-related measurement invariance with Australian cricketers. Psychol Sport Exerc. 2011;12:423-33.

19. Gonzalez SP, Moore EW, Newton M, Galli NA. Validity and reliability of the Connor-Davidson resilience scale (CD-RISC) in competitive sport. Psychol Sport Exerc. 2016;23:31-9.

20. Notario-Pacheco B, Solera-Martínez M, Serrano-Parra MD, BartoloméGutiérrez R, García-Campayo J, Martínez-Vizcaíno V. Reliability and validity of the Spanish version of the 10 -item Connor-Davidson resilience

\section{CONFIDENCIALIDADE DOS DADOS}

Os autores declaram ter seguido os protocolos do seu centro de trabalho acerca da publicação de dados.

\section{CONFLITOS DE INTERESSE}

Os autores declaram não ter conflitos de interesse relacionados com o presente trabalho.

\section{FONTES DE FINANCIAMENTO}

Este projeto não recebeu qualquer apoio financeiro.

scale (10-item CD-RISC) in young adults. Health Qual Life Outcomes. 2011;9:63-8.

21. Blanco V, Guisande MA, Sánchez MT, Otero $P$, Vázquez FL. Spanish validation of the 10-item Connor-Davidson Resilience Scale (CD-RISC 10) with non-professional caregivers. Aging Ment Heal. 2019;23:183-8.

22. Shin GS, Choi KS, Jeong KS, Min YS, Ahn YS, Kim MG. Psychometric properties of the 10-item Conner-Davidson resilience scale on toxic chemical-exposed workers in South Korea. Ann Occup Environ Med. 2018;30:1-7.

23. Soler Sánchez MI, Meseguer de Pedro M, García Izquierdo M Propriedades psicométricas de la versión española de la escala de resiliencia de 10 ítems de Connor-Davidson (CD-RISC 10) en una muestra multiocupacional. Rev Latinoam Psicol. 2016;48:159-66.

24. Wang L, Zhanbiao S, Zhang Y, Zhang Z. Psychometric properties of the 10-item Connor-Davidson resilience scale in Chinese earthquake victims. Psychiatry Clin Neurosci. 2010;64:499-504.

25. Lopes VR, Martins MC. Validação fatorial da escala de resiliência de Connor-Davidson (Cd-Risc-10) para Brasileiros. Rev Psicol Organ e Trab. 2011;2:36-50.

26. Valera MM, Meseguer de Pedro M, Soler Sánchez MI. Propiedades psicométricas de la versión española de la escala de resiliencia de 10 ítems de Connor-Davidson (CD-RISC 10) en una muestra de desempleados. Summa Psicol UST. 2018;15:1-9.

27. Coates EE, Phares V, Dedrick RF. Psychometric properties of the Connor-Davidson resilience scale 10 among low-income, African American men. Psychol Assess. 2013;25:1349-54.

28. Goins RT, Gregg JJ, Fiske A. Psychometric properties of the ConnorDavidson resilience scale with older american indians: the native elder care study. Res Aging. 2013;35:123-43.

29. Ye ZJ, Qiu HZ, Li PF, Chen P, Liang MZ, Liu ML, et al. Validation and application of the Chinese version of the 10-item Connor-Davidson resilience scale (CD-RISC-10) among parents of children with cancer diagnosis. Eur J Oncol Nurs. 2017;27:36-44.

30. Liu DW, Fairweather-Schmidt AK, Burns RA, Roberts RM. The ConnorDavidson resilience scale: establishing invariance between gender across the lifespan in a large community based study. J Psychopathol Behav Assess. 2015;37:340-8.

31. Spera SP, Buhrfeind ED, Pennebaker JW, Buhrfeind ED, Pennebaker JW. Expressive writting and coping with job loss. Acad Manag. 2014;37:722-33.

32. Moorhouse A, Caltabiano ML. Resilience and unemployment: exploring risk and protective influentes for the outcome variables of depression and assertive job searching. J Employ Couns. 2007;44:115-25.

33. Ślebarska K, Moser K, Gunnesch-Luca G, Slebarka K, Moser K, Gunnesch-Luca G. Unemployment, social support, individual resources, and job search behavior. J Employ Couns. 2009;46:159-70.

34. Smith KJ, Emerson DJ, Schuldt MA. A demographic and psychometric assessment of the Connor-Davidson resilience scale 10 (CD-RISC 10) with a US public accounting sample. J Account Organ Chang. 2018;14:513-34.

35. Faria-Anjos J, Ribeiro MT, Ribeiro M. Psychometric evaluation of the Connor-Davidson resilience scale (CD-RISC) in a Portuguese population. Lisbon: Conferência da NATO; 2010.

36. Davidson JR, Connor KM. Resilience Scale (CD-RISC) Manual. 2018 [consultado 2018 jan 14]. Disponível em: http://www.connordavidsonresiliencescale.com/CD-RISC\%20Manual\%2008-19-18.pdf.

37. Cohen J. Statistical power analysis for the behavioral sciences. $2^{\text {nd }}$ ed. Hillsdale NJ, editor. Mahwah: Lawrence Erlbaum Associates Publisher; 
1988.

38. Hu LT, Bentler P. Cutoff criteria for fit indexes in covariance structure analysis: conventional criteria versus new alternatives, structural equation modeling. Struct Equ Model. 1999;6:1-55.

39. Browne MW, Cudeck R. Alternative ways of assessing model fit. Sociol Methods Res. 1992;21:230-58.

40. Cheung GW, Rensvold RB. Evaluating goodness-of-fit indexes for testing measurement invariance. Struct Equ Model A Multidiscip J. 2002;9:233-55.

41. Lee EE, Martin AS, Tu X, Palmer BW, Jeste DV. Childhood adversity and schizophrenia: the protective role of resilience in mental and physical health and metabolic markers. J Clin Psychiatry. 2018;79:17m11776.

42. Ferreira PL, Santana P. Percepção de estado de saúde e de qualidade de vida da população activa: contributo para a definição de normas portuguesas. Rev Port Saúde Pública. 2003;21:15-30.

43. Goldberg DP, Williams $P$, editores. A user's guide to the general health questionnaire. Windsor: Berks NFER-Nelson; 1988.

44. Mclntyre TM, Mclntery SE, Araújo-Soares V, Figueredo M, Johnston D, Faria F. Psychophysiological and psychosocial indicators of the efficacy of a stress managment program for health profissionals. Phase I. In: Cox T, Dewe PK, Cox R, editors. Occupational health psychology Europe 2000. European A. Health Psychology Conference Proceedings Series. Nottingham: I-WHO Publications: 2000. p. 112-6.

45. Overholser BR, Sowinski KM. Biostatistics primer: part 2. Nutr Clin Pract. 2008;23:76-84

46. Nunnally J. Psychometric theory. $2^{\text {nd }}$ ed. New York: McGraw-Hill; 1978.

47. Tabachnick B, Fidell L. Using multivariate statistics. $6^{\text {th }}$ ed. Boston: Pearson Education, Inc; 2013.

48. Schermelleh-Engel $\mathrm{K}$, Moosbrugger $\mathrm{H}$, Müller $\mathrm{H}$. Evaluating the fit of structural equations models: test of significance and descriptive goodness-of-fit measures. Methods Psychol Res Online. 2003;8:23-74.
49. Bentler PM, Bonnet DG. Significance tests and goodness of fit in the analysis of covariance structure. Phychological Bull. 1980;88:588-606.

50. Aloba O, Olabisi O, Aloba T. The 10-Item Connor-Davidson resilience scale: factorial structure, reliability, validity, and correlates among student nurses in Southwestern Nigeria. J Am Psychiatr Nurses Assoc. 2016;22:43-51.

51. Lauridsen LS, Willert MV, Eskildsen A, Christiansen DH. Crosscultural adaptation and validation of the Danish 10-item ConnorDavidson resilience scale among hospital staff. Scand J Public Health. 2017;45:654-7.

52. Tsigkaropoulou E, Douzenis A, Tsitas N, Ferentinos P, Liappas I, Michopoulos I. Greek version of the Connor-Davidson resilience scale: psychometric properties in a sample of 546 subjects. In Vivo. 2018;32:1629-34.

53. Lamond AJ, Depp C, Allison M, Langer R, Reichstadt J, Moore DJ, et al. Measurement and predictors of resilience among community-dwelling older women. J Psychiatr Res. 2008;43:148-54.

54. Jeong HS, Kang I, Namgung E, Im JJ, Jeon Y, Son J, et al. Validation of the Korean version of the Connor-Davidson Resilience Scale-2 in firefighters and rescue workers. Compr Psychiatry. 2015;59:123-8.

55. Duong C, Hurst CP. Reliability and validity of the Khmer version of the 10-item Connor-Davidson resilience scale (Kh-CD-RISC10) in Cambodian adolescents. BMC Res Notes. 2016;9:1-7.

56. McDonald RP, Ho MH. Principles and practice in reporting structural equation analyses. Psychol Methods. 2002;7:64-82.

57. Hogarty KY, Hines CV, Kromrey JD, Perron JM, Mumford AK. The quality of factor solutions in exploratory factor analysis: the influence of sample size, communality, and overdetermination. Educ Psychol Meas. 2005;65:202-26.

58. Davydov DM, Stewart R, Ritchie K, Chaudieu I. Resilience and mental health. Clin Psychol Rev. 2010;30:479-95. 